\title{
Boundary Value Problems for Complete Partial Differential Equations of Variable Order
}

\author{
F. E. Lomovtsev \\ Belarus State University, Minsk, Belarus \\ Received January 26, 2009
}

\begin{abstract}
We prove the well-posedness of new boundary value problems for partially pseudodifferential complete nonclassical equations of variable order in space variables with higher derivatives of odd order in time.
\end{abstract}

DOI: $10.1134 /$ S0012266110090144

Boundary value problems for parabolic equations in variable-order partial derivatives (in space variables) were earlier considered for higher derivatives of the first order [1-3] and odd order [4] in time, for hyperbolic equations with higher derivatives of the second order [3] and even order [5] in time, for partially pseudodifferential parabolic equations with higher derivatives of the first order [6] and higher order [7] in time, and for partially pseudodifferential hyperbolic equations with even-order derivatives in time [8]. In the present paper, we prove the strong well-posedness of new boundary value problems for partially pseudodifferential complete nonclassical equations of variable order with odd-order higher derivatives in time. The variable differentiation order depended on the time in $[1-5]$ and on space points at which the differentiation is performed in $[6,7]$.

\section{STATEMENT OF THE PROBLEM}

In the domain $G=] 0, T\left[\times \mathbb{R}^{n}\right.$, consider the boundary value problems

$$
\begin{aligned}
& (-1)^{m} \frac{\partial^{2 m+1} u(t, x)}{\partial t^{2 m+1}}+\sum_{s=1}^{2 m} \frac{\partial^{[(s+1) / 2]}}{\partial t^{[(s+1) / 2]}}(-1)^{[s / 2]}\left(I-\Delta_{x}\right)^{p_{s}(t)-\varepsilon_{s}} \frac{\partial^{[s / 2]} u(t, x)}{\partial t^{[s / 2]}} \\
& \left.\quad+\lambda_{m}\left(I-\Delta_{x}\right)^{p_{0}(t)} u(t, x)=f(t, x), \quad t \in\right] 0, T\left[, \quad x=\left(x_{1}, \ldots, x_{n}\right) \in \mathbb{R}^{n},\right. \\
& \partial^{i} u(0, x) / \partial t^{i}=\partial^{j} u(T, x) / \partial t^{j}=0, \\
& x \in \mathbb{R}^{n}, \quad i=0, \ldots, m, \quad j=0, \ldots, m-1, \quad m=0,1, \ldots,
\end{aligned}
$$

where $p_{s}(t)=p(t)\{1-(s-1) /(2 m)\}, 0<\varepsilon_{s}<1 /(2 m),[a]$ is the integer part of the number $a$, and the fractional partial derivatives with respect to $x$ are defined with the use of the well-known Fourier-Plancherel (F-P) transforms in $L_{2}\left(\mathbb{R}^{n}\right)$ [9, p. 108]. Here the operators

$$
A_{s}(t) u(x)=(-1)^{[s / 2]} F^{-1}\left[\left(1+|\xi|^{2}\right)^{p_{s}(t)-\varepsilon_{s}} F[u](\xi)\right](x)
$$

with domains $D\left(A_{s}(t)\right)=\left\{u(x) \in L_{2}\left(\mathbb{R}^{n}\right):\left(1+|\xi|^{2}\right)^{p_{s}(t)-\varepsilon_{s}} F[u](\xi) \in L_{2}\left(\mathbb{R}^{n}\right)\right\}, t \in[0, T]$, are pseudodifferential operators of variable order with respect to $x[9, \mathrm{pp} .135-136]$ corresponding to the symbols $a_{s}(t, x, \xi)=\left(1+|\xi|^{2}\right)^{p_{s}(t)-\varepsilon_{s}}, s \geq 0, \varepsilon_{0}=0$ [10, pp. 94-98 of the Russian translation].

\section{EXISTENCE, UNIQUENESS, AND CONTINUOUS DEPENDENCE}

The spaces of strong solutions and right-hand sides are the Hilbert spaces $\mathcal{E}^{m}(G)$ defined as the closures of the sets $D\left(L_{m}\right)$ of all functions $C^{2 m+1, p^{*}}(G)$, where $p^{*}=\left[4 \max _{t \in[0, T]} p(t)\right]+1$, satisfying 
conditions (2) and compactly supported in $\mathbb{R}^{n}$ in the Hermitian norms

$$
\|u\|_{m}=\left\{\int_{0}^{T} \int_{\mathbb{R}^{n}}\left(\left|\frac{\partial d^{m} u(t, x)}{\partial d t^{m}}\right|^{2}+\left|\left(I-\Delta_{x}\right)^{p_{0}(t) / 2} u(t, x)\right|^{2}\right) d x d t\right\}^{1 / 2}
$$

and the Banach spaces $\widehat{\mathcal{F}}^{-m}(G)$ defined as the closures of the set $L_{2}(G)$ in the norms

$$
\langle\|f\|\rangle_{-m}=\sup _{v \in \mathcal{E}^{m}(G)}\left\{\left|\int_{0}^{T}(T-t) \int_{\mathbb{R}^{n}} f \bar{v} d x d t\right|\left(|||v| \|_{m}\right)^{-1}\right\},
$$

respectively. The boundary value problems (1), (2) correspond to linear unbounded operators $L_{m}\left(\lambda_{m}\right): E^{m} \supset D\left(L_{m}\right) \rightarrow \widehat{F}^{-m}$ with dense domains $D\left(L_{m}\right)$.

Definition 1. The solutions of the operator equations $\bar{L}_{m}\left(\lambda_{m}\right) u=f, f \in \widehat{F}^{-m}, m=0,1, \ldots$, are referred to as strong solutions of the boundary value problems (1), (2).

Theorem 1. If $n / 2<p(t) \in C^{m+1}[0, T]$, and the derivative $p^{\prime}(t), t \in[0, T]$, is nonpositive, then there exist $c_{0}(m)>0, \widetilde{\Lambda}_{0}=\left[1,+\infty\left[\right.\right.$ for $m=0$ and $\widetilde{\Lambda}_{m}=\left[\widetilde{\lambda}_{m},+\infty\left[, \widetilde{\lambda}_{m} \geq 1\right.\right.$, for $m>0$, such that, for all $f \in \widehat{\mathcal{F}}^{-m}(G)$ and $\lambda_{m} \in \widetilde{\Lambda}_{m}$, the boundary value problems (1) and (2) have a unique strong solution $u \in C^{m-1}\left([0, T], L_{2}\left(\mathbb{R}^{n}\right)\right) \cap \mathcal{E}^{m}(G)$, and

$$
\|\| u(t, x) \mid \|_{m} \leq c_{0}(m)\langle\| f(t, x)||\rangle_{-m}, \quad m=0,1, \ldots
$$

Proof. The proof amounts to verifying the assumptions of Theorem 2 in [11] for the operators $A(t)=\left(I-\Delta_{x}\right)^{p(t)}$ with domains $D(A(t))=\left\{u(x) \in L_{2}\left(\mathbb{R}^{n}\right):\left(1+|\xi|^{2}\right)^{p(t)} F[u](\xi) \in L_{2}\left(\mathbb{R}^{n}\right)\right\}$ and for the smoothing operators $B_{\varepsilon}^{-1}(t)=(I-\varepsilon B(t))^{-1}, \varepsilon>0, B(t)=-A_{0}(t)$.

I. Maximal accretivity of the operators $A_{0}(t)$. The proof of the self-adjointness and positive definiteness of the operators $A_{0}(t)$ in $L_{2}\left(\mathbb{R}^{n}\right)$ can be found in [8, p. 533].

II. Existence of self-adjoint operators $A(t)$ subordinate to the operators $A_{0}(t)$. By using two basic properties of the F-P transforms, the Parseval relation $\langle h, g\rangle=(2 \pi)^{-n}\langle F[h], F[g]\rangle, h, g \in$ $L_{2}\left(\mathbb{R}^{n}\right)$, for the inner product $\langle\cdot, \cdot\rangle$ in $L_{2}\left(\mathbb{R}^{n}\right)$, and the inversion formulas $F^{-1} F[g]=F F^{-1}[g]=g$, $g \in L_{2}\left(\mathbb{R}^{n}\right)$, we find that inequalities (4) in [11] hold for all $t \in[0, T]$ and $u \in D\left(A_{0}(t)\right)$ :

$$
\begin{aligned}
\operatorname{Re}\left\langle A_{0}(t) u, u\right\rangle & =\operatorname{Re}\left\langle F^{-1}\left[\left(1+|\xi|^{2}\right)^{p_{0}(t)} F[u]\right], u\right\rangle=(2 \pi)^{-n}\left\langle\left(1+|\xi|^{2}\right)^{p_{0}(t)} F[u], F[u]\right\rangle \\
& \geq(2 \pi)^{-n}\left\langle\left(1+|\xi|^{2}\right)^{p(t)} F[u], F[u]\right\rangle=\left\langle F^{-1}\left[\left(1+|\xi|^{2}\right)^{p(t)} F[u]\right], u\right\rangle=\langle A(t) u, u\rangle .
\end{aligned}
$$

Let us compute the strong derivative $d A^{-1}(t) / d t$ of the operators $A^{-1}(t)$ inverse to the operators $A(t)$. If $p(t)>n / 2$, then, by using the continuity of $F^{-1}$ with respect to $t$ in $L_{2}\left(\mathbb{R}^{n}\right)$ and the convolution estimate

$$
\|h * g\|_{L_{2}} \leq\|h\|_{L_{1}}\|g\|_{L_{2}}
$$

[9, p. 62] and by performing the differentiation of the operators $A^{-1}(t) g=F^{-1}\left[\left(1+|\xi|^{2}\right)^{-p(t)}\right] *$ $g \in L_{2}\left(\mathbb{R}^{n}\right)$ with respect to $t$ for all $g \in L_{2}\left(\mathbb{R}^{n}\right)[9$, pp. 182-185], we prove the existence of a strong derivative $\left(d A^{-1}(t) / d t\right) g=-p^{\prime}(t)\left(F^{-1}\left[\left(1+|\xi|^{2}\right)^{-p(t)} \ln \left(1+|\xi|^{2}\right)\right] * g\right)$ in $L_{2}\left(\mathbb{R}^{n}\right)$, and the boundedness of this derivative in $L_{2}\left(\mathbb{R}^{n}\right)$ follows from the inequalities [8, p. 533] $\left\|\left(d A^{-1}(t) / d t\right) g\right\| \leq$ $(\varrho e)^{-1}\left|p^{\prime}(t)\right|\|g\|$, where $\|\cdot\|$ is the norm in $L_{2}\left(\mathbb{R}^{n}\right)$, and $\varrho>0$ is an arbitrarily small number for $i=1$ in the inequalities $\ln ^{i} z \leq(i /(\varrho e))^{i} z^{\varrho}, z \geq 1, \varrho>0, i=1,2, \ldots$ The verification of the corresponding assumption of Theorem 2 for the derivative $d A^{-1}(t) / d t$ can be found in [8, p. 537].

III. Differentiability and symmetry of the operators $A_{s}(t), s>0$. The operators $A(t)$ have positive fractional powers $A^{\alpha /(2 m)}(t) u=F^{-1}\left[\left(1+|\xi|^{2}\right)^{p(t) \alpha /(2 m)} F[u]\right], 0 \leq \alpha \leq 2 m$. The operators $A_{s}(t)$ belong to $\mathcal{B}\left([0, T], \mathcal{L}\left(W^{2 m+1-s}(t), H\right)\right), s>0$, since

$$
\begin{aligned}
& \left\|A_{s}(t) u\right\|^{2}=\left\|F^{-1}\left[\left(1+|\xi|^{2}\right)^{p_{s}(t)-\varepsilon_{s}} F[u]\right]\right\|^{2}=(2 \pi)^{-n}\left\|\left(1+|\xi|^{2}\right)^{p_{s}(t)-\varepsilon_{s}} F[u]\right\|^{2} \\
& \quad \leq(2 \pi)^{-n}\left\|\left(1+|\xi|^{2}\right)^{p_{s}(t)} F[u]\right\|^{2}=\left\|F^{-1}\left[\left(1+|\xi|^{2}\right)^{p_{s}(t)} F[u]\right]\right\|^{2}=|u|_{2 m+1-s, t}^{2} \quad \forall u \in W^{2 m+1-s}(t) .
\end{aligned}
$$

DIFFERENTIAL EQUATIONS $\quad$ Vol. $46 \quad$ No. $9 \quad 2010$ 
The first strong derivative of the linear unbounded operators $A_{s}(t)$ with variable domains $D\left(A_{s}(t)\right)$ in the sense of the definition in [11] was computed in [8, p. 535] and is equal to

$$
\left(d A_{s}(t) / d t\right) u(t)=(-1)^{[s / 2]} c_{m}(s, p) F^{-1}\left[\left(1+|\xi|^{2}\right)^{p_{s}(t)-\varepsilon_{s}} \ln \left(1+|\xi|^{2}\right) F[u(t)]\right],
$$

where $c_{m}(s, p)=(1-(s-1) /(2 m)) p^{\prime}(t)$. In a similar way, by using two basic properties of the $\mathrm{F}-\mathrm{P}$ transforms, one can show that

$$
\begin{aligned}
& \left\|\left(d A_{s}(t) / d t\right) u\right\|^{2} \leq c_{m}^{2}(s, p)(2 \pi)^{-n}\left\|\left(1+|\xi|^{2}\right)^{p_{s}(t)-\varepsilon_{s}+\varrho} F[u]\right\|^{2} \\
& \quad \leq c_{m}^{2}(s, p)(2 \pi)^{-n}\left\|\left(1+|\xi|^{2}\right)^{p_{s}(t)+p(t) \tau /(2 m)} F[u]\right\|^{2} \leq c_{m}^{2}(s, p)|u|_{2 m+1-s+\tau, t}^{2} \quad \forall u \in W^{2 m+1-s+\tau}(t)
\end{aligned}
$$

for an arbitrarily small $\tau>0$; i.e., $d A_{s}(t) / d t \in \mathcal{B}\left([0, T], \mathcal{L}\left(W^{2 m+1-s+\tau}(t), H\right)\right), s>0$, if $0<\varrho \leq$ $\varepsilon_{s}+n \tau /(4 m)$ for $i=1$. Since $p(t) \in C^{m+1}[0, T]$, it follows that the remaining strong derivatives of the operators $A_{s}(t)$ can be evaluated recursively in $j$, and one can show that if $0<\varrho \leq \varepsilon_{s}+n \tau /(4 m)$ for $i=j$, then $d^{j} A_{s}(t) / d t^{j} \in \mathcal{B}\left([0, T], \mathcal{L}\left(W^{2 m+1-s+\tau}(t), H\right)\right), j=2, \ldots,[(s+1) / 2], s>0$.

The operators $A_{s}(t)$ satisfy inequalities (5) in [11] for $j=0,1$; for example, if $j=1$, then

$$
\begin{aligned}
&\left|\left\langle\left(d A_{s}(t) / d t\right) u, v\right\rangle\right|=\frac{\left|c_{m}(s, p)\right|}{(2 \pi)^{n}} \mid\left\langle\left(1+|\xi|^{2}\right)^{p(t)\left(1 / 2-[s / 2] /(2 m)+p_{s} /(2 m)\right)-\varepsilon_{s}}\right. \\
&\left.\quad \times \ln \left(1+|\xi|^{2}\right) F[u],\left(1+|\xi|^{2}\right)^{p(t)\left(1 / 2-[(s-1) / 2] /(2 m)-p_{s} /(2 m)\right)} F[v]\right\rangle \mid \\
& \leq\left|c_{m}(s, p)\right||u|_{m-[s / 2]+p_{s}, t}|v|_{m-[(s-1) / 2]-p_{s}, t} \quad \forall u \in W^{m-[s / 2]+p_{s}}(t), \quad \forall v \in W^{m-[(s-1) / 2]-p_{s}}(t)
\end{aligned}
$$

provided that $0<\varrho \leq \varepsilon_{s}, s>0$, for $i=1$. Here we have used two basic properties of F-P transforms. The proof of the symmetry of the operators $A_{s}(t), s>0$, can be found in [8, p. 535]. Inequalities (6) in [11] hold for the operators $A_{s}(t), s>0$, since

$$
\begin{aligned}
(-1)^{(s+2) / 2}\left\langle A_{s}(t) u, u\right\rangle & =-\left\langle F^{-1}\left[\left(1+|\xi|^{2}\right)^{p(t)(1-(s-1) /(2 m))-\varepsilon_{s}} F[u]\right], u\right\rangle \\
& =-(2 \pi)^{-n}\left\langle\left(1+|\xi|^{2}\right)^{p(t)(1-(s-1) /(2 m))-\varepsilon_{s}} F[u], F[u]\right\rangle \leq 0 \quad \forall u \in D(A(t)) .
\end{aligned}
$$

$I V$. Properties of smoothing operators $B_{\varepsilon}^{-1}(t)$ and the corresponding smoothed operators $A_{s}(t)$, $s>0$. 1. In $L_{2}\left(\mathbb{R}^{n}\right)$, the dissipativity of the operators $B(t)=-A_{0}(t)$ follows from the positive definiteness of the operators $A_{0}(t)$, and the proof of the boundedness of the strong derivatives $d^{i} B^{-1}(t) / d t^{i}=d^{i} B^{*-1}(t) / d t^{i}, i=0, \ldots, m+1$, can be found in [8, p. 534]. For example, for $B^{-1}(t) g=-A_{0}^{-1}(t) g=-\left(F^{-1}\left[\left(1+|\xi|^{2}\right)^{-p_{0}(t)}\right] * g\right)$, the strong derivative is

$$
\left(d B^{-1}(t) / d t\right) g=(1+1 /(2 m)) p^{\prime}(t)\left(F^{-1}\left[\left(1+|\xi|^{2}\right)^{-p_{0}(t)} \ln \left(1+|\xi|^{2}\right)\right] * g\right),
$$

and $\left\|\left(d B^{-1} / d t\right) g\right\| \leq(1+1 /(2 m))\left|p^{\prime}(t)\right|(\varrho e)^{-1}\|g\|$ for all $g \in L_{2}\left(\mathbb{R}^{n}\right)$. The boundedness of the operators $A_{s}(t)\left(d B^{-1}(t) / d t\right)$ in $L_{2}\left(\mathbb{R}^{n}\right)$ follows from the relations

$$
\begin{aligned}
\left\|A_{s}(t)\left(d B^{-1}(t) / d t\right) g\right\|^{2} & =c_{m}^{2}(0, p)\left\|F^{-1}\left[\left(1+|\xi|^{2}\right)^{-p(t) s /(2 m)-\varepsilon_{s}} \ln \left(1+|\xi|^{2}\right) F[g]\right]\right\|^{2} \\
& \leq c_{m}^{2}(0, p)(2 \pi)^{-n}(\varrho e)^{-2}\left\|\left(1+|\xi|^{2}\right)^{\varrho-\varepsilon_{s}} F[g]\right\|^{2} \leq c_{m}^{2}(0, p)(\varrho e)^{-2}\|g\|^{2} \\
& s=0, \ldots, 2 m, \quad \forall g \in L_{2}\left(\mathbb{R}^{n}\right),
\end{aligned}
$$

if $0<\varrho \leq \varepsilon_{s}$ for $i=1$. Here we have used the $\mathrm{F}-\mathrm{P}$ transform of the convolution $F[h * g]=F[h] \cdot F[g]$, $h \in L_{1}\left(\mathbb{R}^{n}\right), g \in L_{2}\left(\mathbb{R}^{n}\right)\left[9\right.$, p. 105]. For the remaining strong derivatives $d^{i} B^{-1}(t) / d t^{i}$, the proof of the boundedness of the operators $A_{s}(t)\left(d^{i} B^{-1}(t)\right) / d t^{i}, i=2, \ldots,[s / 2], s=0, \ldots, 2 m$, in $L_{2}\left(\mathbb{R}^{n}\right)$ can be performed in a similar way with the use of three basic properties of $\mathrm{F}-\mathrm{P}$ transforms.

2. For $m=0$, there exists the first limit in (7) in [11]: if $u \in W^{m+1 / 2}(t)$, then

$$
\begin{aligned}
& \left|\operatorname{Re}\left\langle\left(d B_{\varepsilon}^{-1}(t) / d t\right)\left(B_{\varepsilon}^{-1}(t)\right)^{*} u, v\right\rangle\right|=\left|\operatorname{Re}\left\langle\varepsilon\left(d B^{-1}(t) / d t\right) B(t) B_{\varepsilon}^{-2}(t) u, B(t) B_{\varepsilon}^{-1}(t) u\right\rangle\right| \\
& \quad \leq\left\|\left(d B^{-1}(t) / d t\right) \sqrt{\varepsilon}(-B(t))^{1 / 2} B_{\varepsilon}^{-2}(t)(-B(t))^{1 / 2} u\right\|\left\|\sqrt{\varepsilon}(-B(t))^{1 / 2} B_{\varepsilon}^{-1}(t)(-B(t))^{1 / 2} u\right\| \rightarrow 0
\end{aligned}
$$


as $\varepsilon \rightarrow 0$, since the operators $d B^{-1}(t) / d t$ are bounded in $L_{2}\left(\mathbb{R}^{n}\right),(-B(t))^{1 / 2} u \in L_{2}\left(\mathbb{R}^{n}\right)$, and $\lim _{\varepsilon \rightarrow 0}\left\|\sqrt{\varepsilon}(-B(t))^{1 / 2} B_{\varepsilon}^{-1}(t) g\right\|=0$ for all $g \in L_{2}\left(\mathbb{R}^{n}\right)$, where $(-B(t))^{1 / 2}$ is the square root of the operators $A_{0}(t)$. The validity of inequalities (7) in [11] follows from (3) in [11].

$3(\mathrm{a})$. For $m>0$, the limit (8) in [11] does not exist, but inequality ( $\left.8^{\prime}\right)$ in [11] takes place:

$$
\begin{aligned}
-\operatorname{Re} & \left\langle\left(d B_{\varepsilon}^{-1}(t) / d t\right)\left(B_{\varepsilon}^{-1}(t)\right)^{*} v, v\right\rangle=-\varepsilon \operatorname{Re}\left\langle B_{\varepsilon}^{-1}(t)(d B(t) / d t) B_{\varepsilon}^{-1}(t)\left(B_{\varepsilon}^{-1}(t)\right)^{*} v, v\right\rangle \\
\leq & \varepsilon^{2} c_{m}(0, p)(2 \pi)^{-n}\left\langle\left(1+|\xi|^{2}\right)^{p_{0}(t)} \ln \left(1+|\xi|^{2}\right) F\left[B_{\varepsilon}^{-2}(t) v\right],\left(1+|\xi|^{2}\right)^{p_{0}(t)} F\left[B_{\varepsilon}^{-2}(t) v\right]\right\rangle \leq 0
\end{aligned}
$$

for all $v \in L_{2}\left(\mathbb{R}^{n}\right)$, since $c_{m}(0, p) \leq 0, t \in[0, T]$, by the assumption of Theorem 1 .

By analogy with the transformations (5), we have

$$
\begin{aligned}
\left\|\left(d\left(B_{\varepsilon}^{-1}(t)\right)^{*} / d t\right) v\right\|^{2} & =\left\|\varepsilon B(t) B_{\varepsilon}^{-1}(t) B^{-1}(t)(d B(t) / d t) B_{\varepsilon}^{-1}(t) v\right\|^{2} \\
& \leq\left\|\varepsilon B(t) B_{\varepsilon}^{-1}(t)\right\|^{2}\left\|B^{-1}(t)(d B(t) / d t) B_{\varepsilon}^{-1}(t) v\right\|^{2} \rightarrow 0 \quad \forall v \in W^{1}(t),
\end{aligned}
$$

since $\lim _{\varepsilon \rightarrow 0}\left\|\varepsilon(B(t)) B_{\varepsilon}^{-1}(t)\right\|=0$ and the values

$$
\begin{aligned}
& \left\|B^{-1}(t)(d B(t) / d t) B_{\varepsilon}^{-1}(t) v\right\|^{2} \\
& \quad=\left\|F^{-1}\left[\left(1+|\xi|^{2}\right)^{-p_{0}(t)}\right] * c_{m}(0, p) F^{-1}\left[\left(1+|\xi|^{2}\right)^{p_{0}(t)} \ln \left(1+|\xi|^{2}\right) F\left[B_{\varepsilon}^{-1}(t) v\right]\right]\right\|^{2} \\
& \quad \leq c_{m}^{2}(0, p)\left\|B_{\varepsilon}^{-1}(t) A^{\delta /(2 m)}(t) v\right\|^{2} \leq c_{m}^{2}(0, p)\left\|A^{\delta /(2 m)}(t) v\right\|^{2}<c_{m}^{2}(0, p)|v|_{1, t}^{2} \quad \forall v \in W^{1}(t)
\end{aligned}
$$

are bounded uniformly with respect to $\varepsilon$ provided that $\delta=2 m \varrho / p(t)<1$ for $0<\varrho \leq n /(4 m)$ if $i=1$. Recurrently in $i$ one can show that $\lim _{\varepsilon \rightarrow 0}\left\|\left(d^{j}\left(B_{\varepsilon}^{-1}(t)\right)^{*} / d t^{j}\right) v\right\|=0$ for all $v \in W^{j-1}(t)$ provided that $0<\varrho \leq n /(4 m)$ for $i=j=2, \ldots, m+1$, and therefore,

$$
\lim _{\varepsilon \rightarrow 0}\left\|\left(d^{i} B_{\varepsilon}^{-1}(t) / d t^{i}\right)\left(B_{\varepsilon}^{-1}(t)\right)^{*} v\right\|=0
$$

for all $v \in W^{i-1}(t)$ and for $i=2, \ldots, m+1$.

By virtue of the estimates (6), as $\varepsilon \rightarrow 0$ we have

$$
\begin{aligned}
& \left\|\left(d B_{\varepsilon}^{-1}(t) / d t\right)\left(d\left(B_{\varepsilon}^{-1}(t)\right)^{*} / d t\right) v\right\|^{2} \\
& \leq\left\|\varepsilon B(t) B_{\varepsilon}^{-1}(t)\right\|^{2}\left\|B^{-1}(t)(d B(t) / d t) B_{\varepsilon}^{-1}(t)\left(d\left(B_{\varepsilon}^{-1}(t)\right)^{*} / d t\right) v\right\|^{2} \rightarrow 0
\end{aligned}
$$

for all $v \in W^{1}(t)$, since, by virtue of the estimates $(7)$ and (6) and the commutativity of $A^{\delta /(2 m)(t)}$ with $B(t)$,

$$
\begin{aligned}
& \left\|B^{-1}(t)(d B(t) / d t) B_{\varepsilon}^{-1}(t)\left(d\left(B_{\varepsilon}^{-1}(t)\right)^{*} / d t\right) v\right\|^{2} \leq c_{m}^{2}(p)\left|\left(d\left(B_{\varepsilon}^{-1}(t)\right)^{*} / d t\right) v\right|_{\delta, t}^{2}, \\
& \left|\left(d\left(B_{\varepsilon}^{-1}(t)\right)^{*} / d t\right) v\right|_{\delta, t}^{2} \leq\left\|\varepsilon B(t) B_{\varepsilon}^{-1}(t)\right\|^{2}\left|B^{-1}(t)(d B(t) / d t) B_{\varepsilon}^{-1}(t) v\right|_{\delta, t}^{2} \rightarrow 0, \\
& \left|B^{-1}(t)(d B(t) / d t) B_{\varepsilon}^{-1}(t) v\right|_{\delta, t}^{2} \leq c_{m}^{2}(0, p)\left\|B_{\varepsilon}^{-1}(t) A^{2 \delta /(2 m)}(t) v\right\|^{2}<c_{m}^{2}(0, p)|v|_{1, t}^{2} \quad \forall v \in W^{1}(t),
\end{aligned}
$$

if $2 \delta=2 m \varrho / p(t)<1$ for $0<\varrho \leq n /(4 m)$ and $i=1$. Recurrently with respect to $i^{\prime}$ and $j$, one can show that if $\left(i^{\prime}+j\right) \delta=2 m \varrho / p(t)<i^{\prime}+j-1$ for $0<\varrho \leq n\left(i^{\prime}+j-1\right) /(4 m)$ and for $i=\max \left\{i^{\prime}, j\right\}$, then $\lim _{\varepsilon \rightarrow 0}\left\|\left(d^{i^{\prime}} B_{\varepsilon}^{-1}(t) / d t^{i^{\prime}}\right)\left(d^{j} B_{\varepsilon}^{-1}(t) / d t^{j}\right) v\right\|=0$ for all $v \in W^{i^{\prime}+j-1}(t), j=1, \ldots, m+1-i^{\prime}$, and $i^{\prime}=1, \ldots, m+1$.

3(b). For all $\tilde{v} \in W^{m-k}(t)$, there exists a limit (9) in [11] as $\varepsilon \rightarrow 0$ :

$$
\begin{aligned}
\left|\left\langle A_{2 k+1}(t)\left(d B_{\varepsilon}^{-1}(t) / d t\right)\left(B_{\varepsilon}^{-1}(t)\right)^{*} \tilde{v}, \tilde{v}\right\rangle\right|=\mid\left\langleF ^ { - 1 } \left[\left(1+|\xi|^{2}\right)^{p(t)(1 / 2-k /(2 m))-\varepsilon_{2 k+1}}\right.\right. \\
\left.\left.\times F\left[\left(d B_{\varepsilon}^{-1}(t) / d t\right)\left(B_{\varepsilon}^{-1}(t)\right)^{*} \tilde{v}\right]\right], F^{-1}\left[\left(1+|\xi|^{2}\right)^{p(t)(1 / 2-k /(2 m))} F[\tilde{v}]\right]\right\rangle \mid \\
\leq\left|\left(d B_{\varepsilon}^{-1}(t) / d t\right)\left(B_{\varepsilon}^{-1}(t)\right)^{*} \tilde{v}\right|_{m-k-\delta_{k}^{\prime}, t}|\tilde{v}|_{m-k, t} \rightarrow 0
\end{aligned}
$$

since, by virtue of the commutativity of the operators $A(t)$ with $B(t)$, the estimates (8) hold for $\delta^{\prime}=m-k-\delta_{k}^{\prime}, \delta_{k}^{\prime}=2 m \varepsilon_{2 k+1} / \max _{t \in[0, T]} p(t) \geq 0, k=0, \ldots, m-1, v=\left(B_{\varepsilon}^{-1}(t)\right)^{*} \tilde{v}$, and, by virtue of the estimate $(9)$,

$$
\begin{aligned}
\left|\left(d B_{\varepsilon}^{-1}(t) / d t\right)\left(B_{\varepsilon}^{-1}(t)\right)^{*} \tilde{v}\right|_{m-k-\delta_{k}^{\prime}, t}^{2} & \leq c_{m}^{2}(0, p)\left\|B_{\varepsilon}^{-2}(t) A^{\left(m-k-\delta_{k}^{\prime}+\varrho^{\prime}\right) /(2 m)}(t) \tilde{v}\right\|^{2} \\
& \leq c_{m}^{2}(0, p)\left\|A^{\left(m-k-\delta_{k}^{\prime}+\varrho^{\prime}\right) /(2 m)}(t) \tilde{v}\right\|^{2} \leq c_{m}^{2}(0, p)|\tilde{v}|_{m-k}^{2}
\end{aligned}
$$

DIFFERENTIAL EQUATIONS $\quad$ Vol. $46 \quad$ No. 92010 
provided that $0<\varrho^{\prime} \leq \delta_{k}^{\prime}, k=0, \ldots, m-1, \varrho^{\prime}=2 m \varrho / \max _{t \in[0, T]} p(t) \geq 0$, for $i=1$. In the same way, one can show that

$$
\begin{gathered}
\lim _{\varepsilon \rightarrow 0}\left\langle A_{2 k+1}(t) B_{\varepsilon}^{-1}(t)\left(d\left(B_{\varepsilon}^{-1}(t)\right)^{*} / d t\right) v, v\right\rangle=0 \\
\lim _{\varepsilon \rightarrow 0}\left\langle A_{2 k}(t)\left(d B_{\varepsilon}^{-1}(t)\left(B_{\varepsilon}^{-1}(t)\right)^{*} / d t\right) v, w\right\rangle=0
\end{gathered}
$$

for all $v \in W^{m-k}(t)$, for all $w \in W^{m-k+1}(t)$, and for $k=1, \ldots, m-1$. If $\varepsilon \rightarrow 0$ strongly in $W^{m-k}(t)$, then

$$
\left|\left(d\left(B_{\varepsilon}^{-1}(t)\right)^{*} / d t\right) v\right|_{m-k, t}^{2} \leq\left\|\varepsilon B(t) B_{\varepsilon}^{-1}(t)\right\|^{2}\left|B^{-1}(t)(d B(t) / d t) B_{\varepsilon}^{-1}(t) v\right|_{m-k, t}^{2} \rightarrow 0
$$

by virtue of the estimates (8) and the inequality [which is a consequence of the estimate (9)]

$$
\left|B^{-1}(t)(d B(t) / d t) B_{\varepsilon}^{-1}(t) v\right|_{m-k, t}^{2} \leq c_{m}^{2}(0, p)\left\|B_{\varepsilon}^{-1}(t) A^{(m-k+\delta) /(2 m)}(t) v\right\|^{2}<c_{m}^{2}(0, p)|v|_{m-k+1, t}^{2}
$$

for all $v \in W^{m-k+1}(t), k=1, \ldots, m-1$, provided that $\delta=2 m \varrho / p(t)<1$ for $0<\varrho \leq n /(4 m)$ and for $i=1$. One can readily show that

$$
\lim _{\varepsilon \rightarrow 0}\left|\left(d^{j}\left(B_{\varepsilon}^{-1}(t)\right)^{*} / d t^{j}\right) v\right|_{m-k, t}=0 \quad \forall v \in W^{m-k+1}(t)
$$

if $0<\varrho \leq n /(4 m)$ for $i=j=2, \ldots, k$ and $k=2, \ldots, m-1$;

$$
\lim _{\varepsilon \rightarrow 0}\left|\left(d^{j} B_{\varepsilon}^{-1}(t)\left(B_{\varepsilon}^{-1}(t)\right)^{*} / d t^{j}\right) v\right|_{m-k, t}=0 \quad \forall v \in W^{m-k+1}(t)
$$

if $j \delta=2 m \varrho / p(t)<m-k+1$ for $0<\varrho \leq n(m-k+1) /(4 m)$ and for $j=1,2, k=1, \ldots, m$, and for all $v \in W^{m-k+j-1}(t)$, if $j \delta=2 m \varrho / p(t)<m-k+j-1$ for $0<\varrho \leq n(m-k+j-1) /(4 m)$, $i=j=2, \ldots, k+1$, and $k=2, \ldots, m$. The property $\lim _{\varepsilon \rightarrow 0}\left\|B_{\varepsilon}^{-1}(t) w-w\right\|=0, w \in L_{2}\left(\mathbb{R}^{n}\right)$, and the commutativity of the operators $A(t)$ and $B(t)$ imply the existence of the limits $(7)$ and (8). By virtue of the estimates (4), we have inequalities $\left(12^{\prime}\right)$ from [11]. The limits (13) in [11] exist for all $s=1, \ldots, 2 m$ and are zero for all $\varepsilon>0$. Inequalities (3) correspond to inequalities (14) in [11]. The proof of Theorem 1 is complete.

\section{REFERENCES}

1. Yurchuk, N.I., Mixed Problems for Parabolic Equations of Variable Order, Dokl. Akad. Nauk SSSR, 1982, vol. 265 , no. 1 , pp. 44-47.

2. Lomovtsev, F.E., Abstract Evolution Differential Equations with Discontinuous Operator Coefficients, Differ. Uravn., 1995, vol. 31, no. 7, pp. 1132-1141.

3. Lomovtsev, F.E., Boundary Value Problems for Operator-Differential Equations with Variable Domains of Smooth and Continuous Operator Coefficients, Doctoral (Phys.-Math.) Dissertation, Minsk, 2003.

4. Lomovtsev, F.E. and Yurchuk, N.I., Boundary Value Problems for Operator-Differential Equations with a Variable Domain of the Operator Coefficients, Differ. Uravn., 1991, vol. 27, no. 10, pp. 1754-1766.

5. Lomovtsev, F.E., Cauchy Problems for Quasihyperbolic Factorized Differential Equations with Variable Domains of Discontinuous Operators, Differ. Uravn., 2007, vol. 43, no. 10, pp. 1433-1436.

6. Egorov, Yu.V. and Nguen Min' Chyong, A Problem with a Directional Derivative in S. L. Sobolev Spaces of Variable Order, Differ. Uravn., 1984, vol. 20, no. 12, pp. 2163-2164.

7. Nguen Min' Chyong and Chan Chi K'et, A Nonclassical Boundary Value Problem for a Pseudodifferential Equation of Variable Order, Differ. Uravn., 2008, vol. 44, no. 8, pp. 1142-1143.

8. Lomovtsev, F.E., Boundary Value Problems for Complete Quasihyperbolic Differential Equations with Variable Domains of Smooth Operator Coefficients. II, Differ. Uravn., 2005, vol. 41, no. 4, pp. 527-537.

9. Vladimirov, V.S., Obobshchennye funktsii $v$ matematicheskoi fizike (Generalized Functions in Mathematical Physics), Moscow: Nauka, 1976.

10. Hörmander, L., The Analysis of Linear Differential Operators, Heidelberg, Springer-Verlag, 1983. Translated under the title Analiz lineinykh differentsial'nykh operatorov s chastnymi proizvodnymi, Moscow: Mir, 1987, Vol. 3.

11. Lomovtsev, F.E., Boundary Value Problems for Complete Quasiparabolic Differential Equations of Odd Orders with Variable Domains of Operators, Differ. Uravn., 2008, vol. 44, no. 6, pp. 841-844. 